\title{
Models of winner and loser effects: a cost-benefit analysis
}

\author{
Karlo Hock $^{1)}$ \& Robert Huber ${ }^{2,3)}$ \\ $\left({ }^{1}\right.$ Division of Molecular Medicine, Rudjer Boskovic Institute, 10000 Zagreb, Croatia; \\ ${ }^{2}$ Department of Biological Sciences, J.P. Scott Center for Neuroscience, Mind \& Behavior, \\ Bowling Green State University, Bowling Green, OH 43403, USA)
}

(Accepted: 19 May 2008)

\begin{abstract}
Summary
Winner and loser effects, in which the probability of future success depends on individual's interaction history, help shape the structure of social hierarchies in animal groups. While reports have documented that both magnitude and symmetry of these effects vary widely across different systems, questions remain whether these effects serve to reduce the associated costs during hierarchy formation. In a series of models, cost-benefit properties of an emerging hierarchy were assessed in relation to variation in winner and loser effects. Coupling high winner effects with low loser effects resulted in an overall increase in aggressiveness in the group, increasing the costs of hierarchy formation for the participants and disrupting its maintenance. In contrast, low winner and high loser effects resulted in an efficient cost reduction while also encouraging stability in social status.
\end{abstract}

Keywords: winner-loser effects, dominance hierarchy, cost-benefit analysis, social rank, aggression.

\section{Introduction}

Winner and loser effects describe situations where the outcome of prior interactions affects future success. Central to the development of dominance relationships among individuals, such effects usually do not alter an individual's actual physical fighting ability, but rather affect its assessment of

\footnotetext{
3) Corresponding author's e-mail address: rh.bgsu@gmail.com 
probabilities for future outcomes or its aggressive motivation. Moreover, experiences associated with each additional interaction appear to be cumulative (Hsu \& Wolf, 2001). These changes in aggressive motivation, such as willingness to engage opponents or timing of the decision to retreat, are based on the receiver's perception of either emitted winner/loser signals, or of its own fighting prowess through self-assessment (Rutte et al., 2006). In either case, winner and loser effects manifest themselves as changes in future success; a winner becomes more likely to win again, while the reverse is true for the loser. With an individual's fighting behavior (e.g., approach and display in the early stages of an encounter) contingent on its own past history, the need for iterated interactions between both familiar and unfamiliar individuals is reduced (Hsu \& Wolf, 1999).

Empirical evidence for the importance of winner and loser effects in the establishment of complex dominance relationships derives from a wide range of taxa (e.g., Hack, 1997; Schuett, 1997; Whitehouse, 1997; Beaugrand \& Goulet, 2000; Huber et al., 2004; Hsu et al., 2006). Moreover, such effects have been the focus of theoretical models and simulations (Bonabeau et al., 1996; Mesterton-Gibbons, 1999; Hemelrijk, 2000, 2002; Beacham, 2003; Dugatkin \& Earley, 2003; Hock \& Huber, 2006, 2007). While the presence of winner and loser effects is widespread, the mechanisms that underlie them are still not adequately understood. Though a significant portion of published studies has focused on dyadic encounters, an increasing body of work is considering the importance of winner and loser effects in the emergence and maintenance of dominance hierarchies in larger group scenarios (e.g., Chase \& Rohwer, 1987; Hemelrijk, 2000; Dugatkin \& Earley, 2003; Dugatkin \& Druen, 2004). In general, the relative magnitude of loser effects often exceeds that of winner effects and is frequently longer-lasting (Francis, 1988; Chase et al., 1994; Schuett, 1997; Bergman et al., 2003). Moreover, loser effects by themselves are often sufficient for maintaining stable hierarchical relationships and social structure (Dugatkin, 1997; Hock \& Huber, 2006, 2007). Current evidence suggests that proximate causes for winner and loser effects reside in behavioral-neuroendocrine feedback loops of aggression (Huber \& Delago, 1998; Huber, 2005; Oyegbile \& Marler, 2005).

The tradeoff between costs and benefits of fighting lies at the very heart of game theoretical analyses (Maynard Smith \& Parker, 1976; Maynard Smith, 1982). It, thus, follows that if winner and loser effects are adaptive, they will contribute to maximization of this ratio. In social context, hierarchies act as 
an effective mechanism to translate social status into differential access to resources (Clutton-Brock et al., 1979; Enquist et al., 1985; Enquist \& Leimar, 1987; Thorpe et al., 1995; Pagel \& Dawkins, 1997; Neat et al., 1998; Cant et al., 2006) and, therefore, both dominant and subordinate individuals will aim to maximize their respective benefit/cost ratios. It is possible to partition the costs of fighting into several categories, such as all costs associated with energy demands of fighting, the risks of injury, and the increased exposure to predation. The benefits of fighting generally focus on resource acquisition, but as many animals fight even in the absence of any obvious resources, such as food or mates, an increased winning probability may represent the resource in and of itself. Consistent with laboratory studies of animal aggression (e.g., Jackson, 1991; Chase et al., 1994; Hsu et al., 2006), winning may effectively serve to prime the agent towards acquisition of resources in the future.

Decapod crustaceans present the researchers with a model system in which animals fight for position even in the absence of resources both in the laboratory (Issa et al., 1999; Goessman et al., 2000; Huber et al., 2004) and under field conditions (Davis \& Huber, 2007). In addition to frequent agonistic interactions, various aspects of winner and loser effects have been documented in Decapoda, from experimental manipulation of loser effects (Huber \& Kravitz, 1995) to their temporal dynamics (Bergman et al., 2003). Decapod crustaceans, thus, present an ideal system for developing theoretical models in order to explore such phenomena.

Our previous work on the relationships between the relative magnitude of winner-loser effects and the formation and maintenance of hierarchical structures (Hock \& Huber, 2006, 2007) has demonstrated that the magnitude of winner-loser effects was important for rapid divergence and hierarchical structuring of ordered ranks, while relatively large loser effects were essential for its long-term stability. In the present study, through a series of spatially explicit agent-based models with strong links to empirical studies, we aim to further explore relationships between winner-loser effects and dominance hierarchies by considering these effects within the context of fighting costs as well as benefits for individuals involved in hierarchy formation. By exploring models which independently vary winner and loser effects, this study aims to shed new light on the general utility of these effects in animal conflicts. 


\section{Methods}

The models were developed using a modeling framework (Hock \& Huber, 2006) integrated into the JavaGrinders library available on the Internet at http://caspar.bgsu.edu/ $\sim$ software/Java/ and the Java programming language (Java 1.6.0 API).

\section{Non-behavioral parameters}

The general parameters were developed so as to closely resemble empirical experiments on decapod hierarchies (Goessmann et al., 2000). The arena, $270 \times 180$ pixels in size (an equivalent of $0.6 \times 0.4 \mathrm{~m}$ of actual space), was implemented as a 'wrap-around' world (where animals exiting one side border would reenter the area from the opposite side), thus allowing for spatial movement patterns unaffected by obstacles. Four individuals were spatially represented in the arena as a single central pixel. A circle surrounding the central pixel, with a diameter of 27 pixels (an equivalent of $6 \mathrm{~cm}$ and twice the carapace length of individuals used in Goessmann et al. (2000), i.e., in line with the experimental thresholds using the critical 'one body length'), was used as a critical distance (DIST) that determined whether two individuals were close enough to interact. At the start of each trial individuals were randomly placed in the arena. Movements for each individual were selected at random from a distribution of 9765 moves that had been recorded in 4-s intervals in freely moving crayfish (Panksepp \& Huber, 2004). Each iteration of the model, thus, represented a 4-s timeframe. Each trial was run for 2700 iterations, or an equivalent of $3 \mathrm{~h}$. No additional rules that would precondition spatial structure or aggregation were implemented in the model.

\section{Interaction rules}

The basic interaction rules were adapted from Hemelrijk (2000). In addition to spatial position, each individual was also characterized by a variable DOM representing its aggressive motivation and, as such, its relative probability of winning in an interaction. Each individual was given a DOM value of 0.5 at the start of each trial, thus leveling the chances to attain any given rank within the group. The cutoff value for minimum DOM was set at 0.0001 . Whenever two individuals came within a DIST of each other, their encounter developed into an interaction with associated consequences for their future 
success. Therefore, each individual was viewed as taking every opportunity towards increasing its own rank. The winner of an interaction was determined by comparing the DOM values of the two contestants, $i$ and $j$, as per equation (1).

$$
p_{\mathrm{W}}=\mathrm{DOM}_{\mathrm{i}} /\left(\mathrm{DOM}_{\mathrm{i}}+\mathrm{DOM}_{\mathrm{j}}\right)
$$

An individual $\mathrm{i}$ won over the individual $\mathrm{j}$ if its probability of winning $p_{\mathrm{W}}$ was greater than a randomly selected number from 0 to 1 . Once the outcome of an interaction was determined, the aggressive motivation of each contestant was updated to reflect it as per equations (2) and (3).

$$
\begin{aligned}
\operatorname{DOM}_{\mathrm{i}(T+1)} & \\
= & \operatorname{DOM}_{\mathrm{i}(T)}+\left(w_{\mathrm{i}}-\left(\operatorname{DOM}_{\mathrm{i}(T)} /\left(\mathrm{DOM}_{\mathrm{i}(T)}+\mathrm{DOM}_{\mathrm{j}(T)}\right)\right)\right) \\
& * \mathrm{STEPDOM}_{\mathrm{DOM}_{\mathrm{j}(T+1)}} \\
= & \operatorname{DOM}_{\mathrm{j}(T)}-\left(w_{\mathrm{i}}-\left(\mathrm{DOM}_{\mathrm{i}(T)} /\left(\mathrm{DOM}_{\mathrm{i}(T)}+\mathrm{DOM}_{\mathrm{j}(T)}\right)\right)\right) \\
& * \mathrm{STEPDOM}
\end{aligned}
$$

If contestant $i$ was a winner, $w_{i}$ was set to 1 , else it was set to 0 . The loser of an interaction would use an escape behavior instead of its next regular move by moving between one and three DIST values directly away from the winner.

From equations (2) and (3) it follows that the subsequent changes in aggressive motivation were dependent not only on the outcome alone, but also on the relative difference in aggressive motivation between contestants and an additional scaling factor STEPDOM. The likelihood of attaining any given intensity level is associated with the fight duration (Huber et al., 2004), and intensity levels in turn have differential consequences on future fighting success (Beaugrand \& Goulet, 2000; Jennings et al., 2005). In general, stereotyped, escalated encounters progress through distinct stages of ritualized assessment and combat; increasingly risky fighting behaviors emerge the longer the fight lasts. In the present models, fight duration was not modeled directly, but rather estimated as a level of aggressive motivation of an individual with a lower DOM value in a pair. While all interactions were resolved instantly in model time and before the next move, the timing of the decision to retreat was calculated by comparing the DOM values of the two 
contestants, selecting a lower one, and then using it to calculate the likelihood of reaching respective fight intensities for a given fight.

In accordance with the previously published characterization of decapod fights (Huber \& Kravitz, 1995; Huber et al., 2004), four discrete intensity levels have been implemented in the model. Different values of STEPDOM were assigned to fights that reached different intensities, where each level $(0$ to 3 ) doubled the previous one. A number of empirical crayfish studies were pooled to obtain probability density functions (logistic curves) describing the association between fight intensity and duration (Huber \& Delago, 1998; Stocker \& Huber, 2001; Schroeder \& Huber, 2002). For each encounter of a given duration the model randomly chose actual fight intensity from among the relevant proportional distributions.

The magnitude of winner and loser effects gradually wears off with time. In Decapoda, winner effects have decayed to about $2 / 3$ of the initial magnitude after a period of 40 min (Bergman et al., 2003). Equation (4) indicates how this decay in DOM values was implemented in the model, with $N$ representing the total number of 4-s intervals.

$$
\mathrm{DOM}_{\text {decayed }}=\mathrm{DOM}_{(\mathrm{t})}-\left(\mathrm{DOM}_{(\mathrm{t})}-0.5\right) /(3 N)
$$

Any changes in DOM value as a result of interactions would eventually return to the resting value if no further interactions took place, with equalsized decay for both winner and loser effects.

\section{Winner and loser effects and cost-benefit analysis}

Both winner and loser effects were modeled implicitly as context-dependent changes in aggressive motivation depending on past interaction outcome. Their manipulation was performed through the use of STEPDOM, thus making it possible to give different values not only to each intensity level, but also to independently combine different sets of STEPDOM values to simulate different magnitudes of winner and loser effects. Two different sets of STEPDOM values were used (High vs. Low) where High set was four times greater than Low set (Table 1). By independently combining these sets for winner and loser effects, four different scenarios, each with 50 replicates, were explored: High winner-Low loser effects (HL), Low winner-High loser effects (LH), High winner-High loser effects (HH), and Low winner-Low loser effects (LL). It was, thus, possible to simulate and, therefore, explore 
Table 1. A list of STEPDOM values used for different intensities in the two sets. The sets of values were independently assigned to either winner or loser effect.

\begin{tabular}{llc}
\hline Intensities & \multicolumn{2}{c}{ STEPDOM } \\
\cline { 2 - 3 } & Low set & High set \\
\hline 0 & 0.03125 & 0.125 \\
1 & 0.0625 & 0.25 \\
2 & 0.125 & 0.5 \\
3 & 0.25 & 1 \\
\hline
\end{tabular}

High set was four times the magnitude of the low set.

how differential magnitudes of winner and loser effects and their relative differences influence the costs and benefits of fights and hierarchy formation within a group.

Measurements of the social status of individuals were taken in regular intervals of 20 interactions within the group regardless of the identity of the involved individuals. The probability to win at a particular point in time was used as a measure of the benefit of fighting. As each individual had an approximately equal chance to meet any of the other three individuals as its next opponent, it was calculated by dividing the pooled $p_{\mathrm{W}}$ values for that individual by three. Each individual's cost of fighting was estimated in two ways: as an average duration of fights and as a percentage of fights that reached the highest intensity for the past 20-interaction interval. Changes in these parameters were classified according to the individual's final ordinal rank. The partition of parameters, coupled with the independent manipulation of winner and loser effects, allowed for a detailed examination of the factors involved in hierarchy formation and how they are influenced by changes in aggressive motivation and interaction history.

Rank stability was determined by examining the probability that the dominant (i.e., highest-ranking) or subordinate (i.e., lowest ranking) individuals of the first 20-interaction interval will either retain, change, or invert their status in the fifth interval (from 80 to 100 interactions). Since our previous work has indicated that disproportionally low loser effects exert a disruptive pressure on hierarchical structure (Hock \& Huber, 2006), a replicated goodness of fit test (Sokal \& Rohlf, 1981) was performed to determine whether loser effects influence the stability of the hierarchy. 


\section{Results}

The social status of an individual was determined by recording its cardinal rank using the Batchelder-Bershad-Simpson (BBS) method (Jameson et al., 1999). Except for determining rank stability, where cardinal ranks were determined on an interval basis, cumulative interaction history was used as the basis for determining cardinal ranks in all other analyses. Individuals that attained the highest cardinal rank in their respective group ( $\alpha$ individuals) were designated as dominants, while those who had the lowest cardinal rank ( $\delta$ individuals) were designated as subordinates. The degree of linearity in hierarchical group structure was measured by Landau's statistic $h$, which compares the number of transitive triads within a group to those maximally possible (Appleby, 1983).

A total of 1431620 -interaction intervals were obtained across the four model groups, with a range of 520-720 and a median value of 640 interactions per group. The number of interactions did not differ among scenarios. These results of interaction frequency when fights are obligate are consistent with the observations reported by Issa et al. (1999), where individuals that took every chance to interact did so at a rate of approx. 250 interactions per hour. However, to facilitate the cross-comparison with our previous work (Hock \& Huber, 2007) we have used only the initial 100 interactions for subsequent analyses.

The degree of linearity approached 1 (perfectly linear) in all models regardless of the magnitude, or the relative magnitude, of winner and loser effects (Figure 1). However, linearity was achieved sooner in the models which had a higher magnitude of loser effects. The linearity results were consistent with those of Hock \& Huber (2006).

The winning probability for an individual was primarily affected by the magnitude of loser effects, with low values producing less of a difference in winning probability among dominants and subordinates (Figure 2). In the HL scenario winning probabilities for both dominants and subordinates remained constant, while the LH scenario produced constant winning probability only for subordinates. The difference in winning probability between dominants and subordinates increased steadily in all scenarios except the HL scenario, while becoming more pronounced with an increase in the relative importance of loser effects.

Average fight duration decreased for both dominants and subordinates in all scenarios except HL, where the fights rapidly reached a maximum (Fig- 

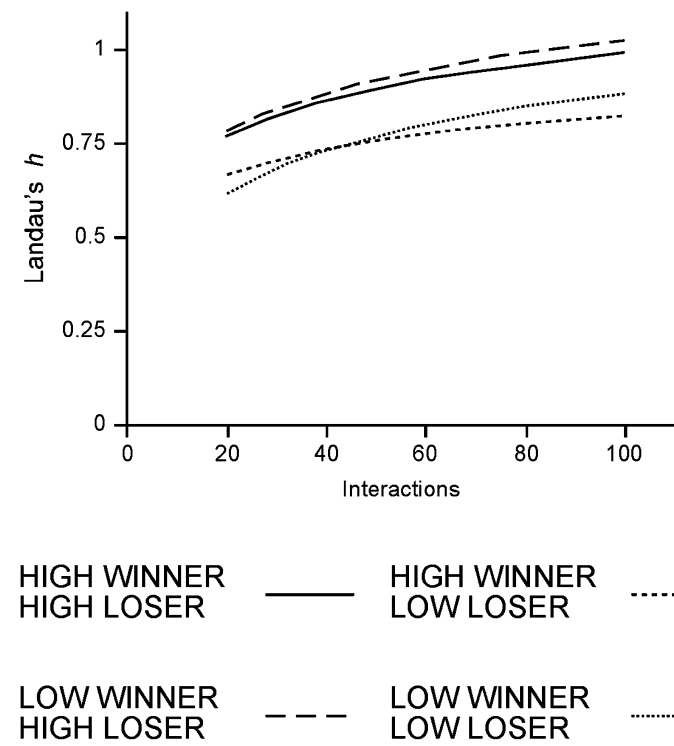

Figure 1. A measure of linearity (Landau's statistic $h$ ) as a function of the number of interactions that have occurred in a group. Linearity of the hierarchical structure increases with subsequent interactions. Models with high magnitude of loser effects exhibit greater linearity for a given number of interactions and also converge more rapidly toward perfect linearity (a $h$ value of 1$)$.

ure 3). Once at a maximum, fight duration then remained constant, indicating that the aggressive motivation of the subordinates remained at high levels with subsequent interactions. In cases where fight duration did decline, the speed of this decline was linked to the relative importance of loser effects (LL scenario had longer fights than HH scenario, and HH scenario had longer fights than LH scenario).

The high level of aggressive motivation across the group in the HL scenario was also evident in the high number of intense fights (Figure 4). A high frequency of intense fighting was observed for both dominants and subordinates in the HL scenario that continued to rise as hierarchies formed, while other scenarios featured low rates of interactions at the highest intensity.

The disruptive influence of the less prominent loser effects on the stability of the hierarchy was examined as the frequency at which the initially dominant or subordinate individuals retained, changed, or completely inverted their status following the initial establishment of dominance status (Table 2). The results of replicated goodness of fit analyses suggest that less prominent loser effects exert a disruptive influence on the maintenance of hierarchical 

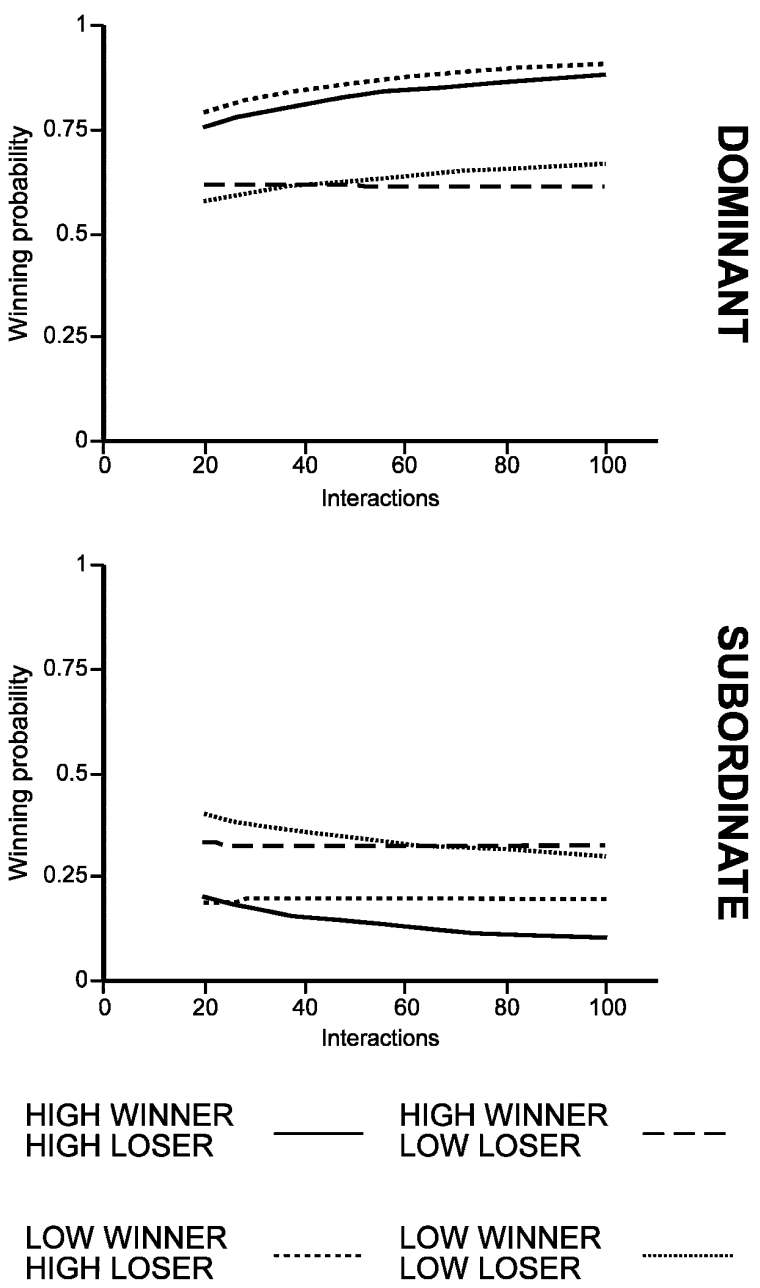

Figure 2. Winning probability as a function of the number of interactions that have occurred in a group. Low winner-High loser scenario results in rising winning probability for dominants and constant winning probability for subordinates.

structure, and, implicitly, on the reliability of initial fighting success as a predictor of eventual social status. Although all scenarios deviated from ideally conserved hierarchical structure, those with a lower magnitude of loser effects exhibited a significantly stronger tendency to do so. For a given magnitude of loser effects, the magnitude of winner effects did not significantly contribute to the stability of the hierarchy. The impact of loser effects was symmetrical for both dominants and subordinates, where this instability of 

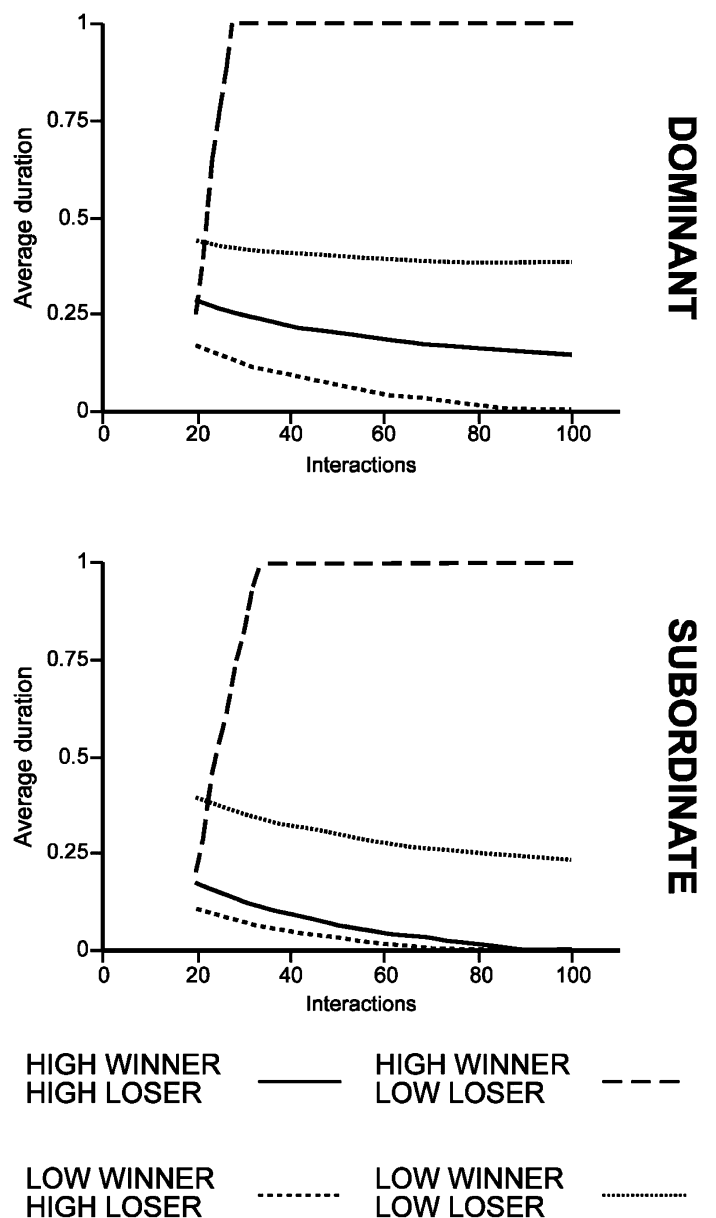

Figure 3. Changes in average duration of fights as a function of the number of interactions that have occurred in a group. High winner-Low loser scenario results in long fights as the aggressive level of the group rises, while Low winner-High loser scenario results in the lowest fight durations overall.

social hierarchy affected the potential of the subordinates to change their position on the hierarchical ladder and also exerted pressure on the dominants striving to retain their preeminence.

\section{Discussion}

The importance of winner and loser effects for resolving animal conflicts has been the focus of many experimental studies and theoretical models (for 

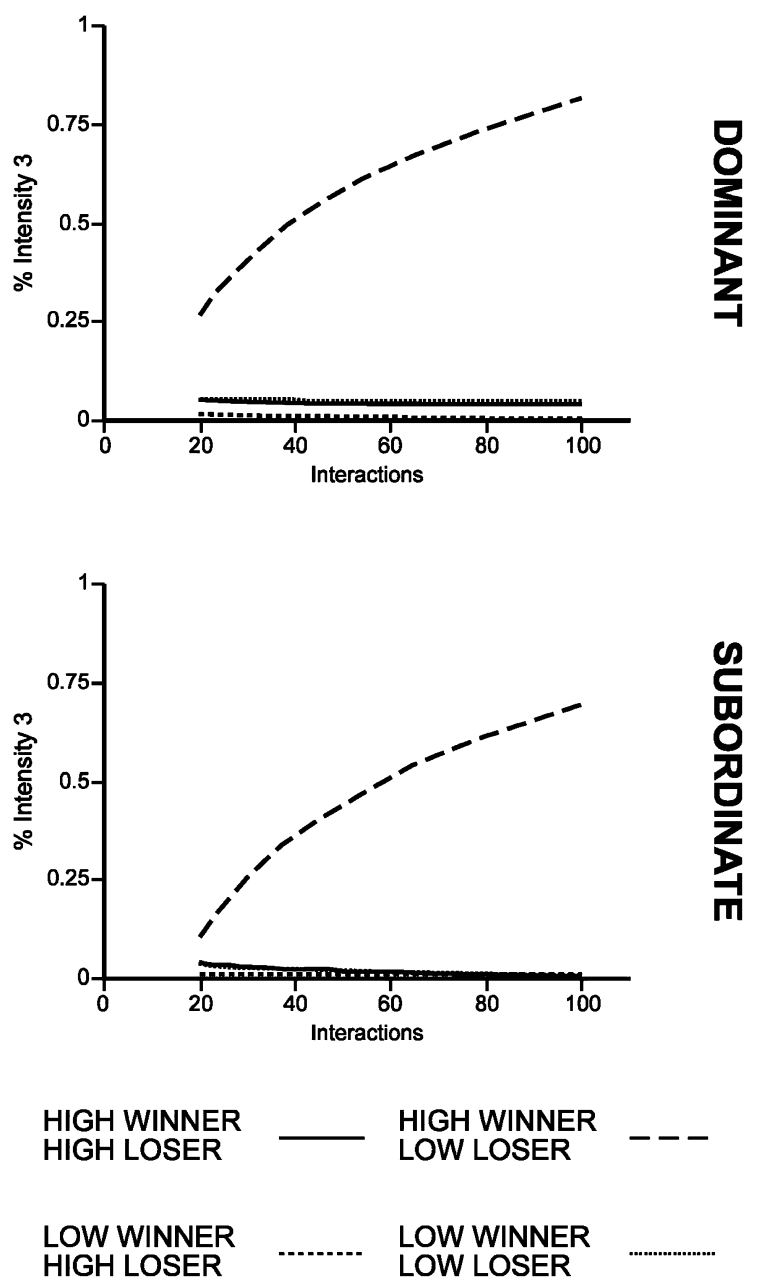

Figure 4. A percentage of fights that ended at maximum intensity of 3 as a function of the number of interactions that have occurred in a group. As the fights progress and the hierarchy is formed, the percentage of fights that reach the highest maximum intensity is rising only in High winner-Low loser scenario.

a review, see Hsu et al., 2006). These effects are the result of a series of decisions that an individual makes in agonistic interactions that ultimately affect its motivation and behavior. As behavioral conventions, they enable the individuals to maximize their access to resources while also retaining the ability to secure resources in future (Hsu \& Wolf, 1999; Mesterton-Gibbons, 1999; Van Doorn et al., 2003). The ability to secure future resources through cost 
Table 2. Results for replicated goodness of fit tests ( $G$-statistic) assessed the impact of loser and winner effects on the stability of hierarchical structure.

\begin{tabular}{|c|c|c|c|c|c|c|c|}
\hline \multirow[t]{2}{*}{ Source } & \multirow[t]{2}{*}{ Initial status } & \multicolumn{3}{|c|}{ Final status } & \multirow[t]{2}{*}{$\mathrm{df}$} & \multirow[t]{2}{*}{$G$} & \multirow[t]{2}{*}{$p$} \\
\hline & & $\alpha$ & $\beta / \gamma$ & $\omega$ & & & \\
\hline \multicolumn{8}{|l|}{ Low loser effect } \\
\hline \multirow{2}{*}{ HL } & $\alpha$ & 16 & 27 & 7 & 2 & 425.818 & $* * *$ \\
\hline & $\omega$ & 8 & 24 & 18 & & & \\
\hline \multirow[t]{2}{*}{ LL } & $\alpha$ & 21 & 23 & 6 & 2 & 399.259 & $* * *$ \\
\hline & $\omega$ & 8 & 26 & 16 & & & \\
\hline$\times$ L total & & & & & 4 & 825.077 & $* * *$ \\
\hline \multirow[t]{2}{*}{$\times \mathrm{L}$ pooled } & $\alpha$ & 37 & 50 & 13 & 2 & 823.839 & $* * *$ \\
\hline & $\omega$ & 16 & 50 & 34 & & & \\
\hline \multicolumn{2}{|l|}{$\times \mathrm{L}$ heterogeneity } & & & & 2 & 1.238 & ns \\
\hline \multicolumn{8}{|l|}{ High loser effect } \\
\hline \multirow[t]{2}{*}{$\mathrm{HH}$} & $\alpha$ & 30 & 17 & 3 & 2 & 239.719 & $* * *$ \\
\hline & $\omega$ & 1 & 21 & 28 & & & \\
\hline \multirow[t]{2}{*}{ LH } & $\alpha$ & 28 & 17 & 5 & 2 & 325.171 & $* * *$ \\
\hline & $\omega$ & 3 & 28 & 19 & & & \\
\hline \multirow{3}{*}{$\begin{array}{l}\times \mathrm{H} \text { total } \\
\times \mathrm{H} \text { pooled }\end{array}$} & & & & & 4 & 564.890 & $* * *$ \\
\hline & $\alpha$ & 58 & 34 & 8 & 2 & 560.870 & $* * *$ \\
\hline & $\omega$ & 4 & 49 & 47 & & & \\
\hline \multicolumn{2}{|l|}{$\times \mathrm{H}$ heterogeneity } & & & & 2 & 4.020 & ns \\
\hline \multicolumn{8}{|l|}{ Totals } \\
\hline \multirow{3}{*}{$\begin{array}{l}\text { Total } \\
\text { Pooled }\end{array}$} & & & & & 8 & 1389.967 & $* * *$ \\
\hline & $\alpha$ & 95 & 84 & 21 & 2 & 1367.065 & $* * *$ \\
\hline & $\omega$ & 20 & 99 & 81 & & & \\
\hline \multicolumn{2}{|l|}{ Heterogeneity } & & & & 6 & 22.902 & $* * *$ \\
\hline \multicolumn{2}{|c|}{ Heterogeneity within $\times \mathrm{H}$ and $\times \mathrm{L}$} & & & & 4 & 5.258 & ns \\
\hline \multicolumn{2}{|c|}{ Heterogeneity between $\times \mathrm{H}$ and $\times \mathrm{L}$} & & & & 2 & 17.640 & $* * *$ \\
\hline
\end{tabular}

Matrices for the individual scenarios were compared to an expected matrix for an ideally conserved hierarchy $\{\{50,0,0\}\{0,0,50\}\}$. The $G$-statistic was used to measure the degree to which the observed values deviated from this hypothesis. It is additive and can be partitioned into separate components for the fit of individual samples, the fit of all samples combined (i.e., pooled fit), and the differences in fit between samples (i.e., heterogeneity). $G$-values can only be obtained for non-zero cells, and a value of +0.5 was, thus, added to every cell of the matrix. All individual scenarios deviated significantly from an ideally conserved social hierarchy. Significant heterogeneity between high $(\times H)$ and low loser effects $(\times L)$ demonstrated that larger loser effects increased hierarchy stability. Winner effects did not significantly contribute to the patterns of this deviation. 
reduction is immanently more important for subordinates who may lack them at present. This may explain why subordinates more strongly abide by social conventions, as evidenced by the number of studies where loser effects exceeded winner effects (Chase et al., 1994), to a point that sometimes winner effects were not detected at all (e.g., Schuett, 1997). Additional mechanisms, such as ritualized fighting with sequential assessment (Enquist \& Leimar, 1983), further minimize fighting costs, particularly if the combatants may face serious injury.

On a group level, winner and loser effects translate into a dominance hierarchy, and such social structuring reduces the need to continuously test the resource acquisition ability of others (Issa et al., 1999; Goessmann et al., 2000). Thus, while the subordinates minimize their own fighting costs through the expression of loser effects, the fighting costs of dominants is also indirectly reduced since they will have to expend less energy for fighting and enjoy lower risks while retaining their preferential access to current resources. The relative magnitude of winner and loser effects should, thus, have a profound effect on the costs and benefits of hierarchical relationships.

In our previous work, winner and loser effects were considered either as mechanisms through which the hierarchies are formed from initially egalitarian groups and maintained over time (Hock \& Huber, 2006), or as indirect consequences of decision-making processes which affect the dynamics of individual fights (Hock \& Huber, 2007). However, the results of the present study shift the focus from the individual's ability to achieve and maintain certain social status to the utility of it doing so. While the general reasoning behind the formation of a hierarchy may be to reduce the risks of future conflicts, this was not the case for all examined scenarios. Specifically, when loser effects were lower than winner effects, the costs of fighting actually increased both in terms of longer fight durations, i.e., energy expenditure, and the intensity of fights, i.e., injury risks. Under such conditions, the aggressive motivation of the subordinates continued to increase despite the fact that they were losing many interactions. This was a result of the combination of two effects. Firstly, low loser effects translated into a relatively small overall decrease in aggressive motivation for the subordinates, even though those losses were frequent. The subordinates, therefore, remained poised to interact in longer fights and at higher intensities. Secondly, this tendency to interact at higher intensities subsequently meant that each win that the subordinates managed to achieve was likely to strongly propel their aggressive 
motivation into positive, thus overshadowing the effect of even a series of losses. Such wins were not only played out at a high intensity, therefore having inherently larger returns and against opponents whose aggressive motivation was also relatively high, but also emphasized by the greater magnitude of winner effects. The winning probability of the subordinates was also comparatively high and constant and, therefore, under these conditions the subordinates were more likely to achieve these wins than in other scenarios. But, even without taking this effect into consideration and though the subordinates were poised to lose fights against the individuals of the highest rank, they were also interacting with opponents against whom their chances of winning were better. Thus, it was not necessary for the subordinates to win against the dominants to achieve these leaps in aggressive motivation, but rather any win was sufficient to attain such result. The constant winning probability under HL conditions, coupled with a less pronounced difference in winning probability between dominants and subordinates, could also explain the eventual convergence of ranks observed in the authors' previous study on the relationships between hierarchical structure and relative magnitude of winner-loser effects (Hock \& Huber, 2006), where disproportionally low loser effects caused the difference among ranks to decrease over time, thus making the gap between individuals of opposite status less explicit.

The HL scenario, therefore, results in highly aggressive individuals at both ends of the hierarchical ladder. There is very little difference between the fight dynamics typically experienced by the dominants and subordinates, with fights being markedly more demanding and risky than those in other scenarios. Furthermore, winning probabilities of both dominants and subordinates remain constant, where hierarchical structures show little reinforcement from subsequent interactions. Fighting history does little to influence the potential for future success beyond the initial divergence and, thus, fights not only become more costly, but also have less significance on the maintenance of social structure. However, such conditions may still prove advantageous for subordinates under certain circumstances, such as in cases where the subordinates have no alternative but to obtain resources (Grafen, 1987) or as a tactic to coerce the dominants into avoiding costly fights (Morrell et al., 2005). If potential benefits outweigh even the most extreme costs incurred by fighting, disproportionally low loser effects may aid the subordinates in destabilization of the hierarchical structure and any attempts to usurp dominant status. 
The increase in the relative magnitude of loser effects is an effective mechanism to reduce the overall costs of fighting, and is consistent with empirical studies that found more prominent loser effects (e.g., Francis, 1988; Bakker et al., 1989; Schuett, 1997; Bergman et al., 2003; but see Hsu \& Wolf, 1999). Fight duration, and to some extent fight intensity, drops as loser effects become more important, and are the lowest in LH scenario. Under such conditions, the individuals that lose fights are rapidly profiled into subordinates, and at the same time lack the mechanism that would advance them to dominance, e.g., with an occasional win against other low-ranking individuals, due to the disproportionally low winner effects. In effect, the mechanism that keeps the subordinates from becoming dominant is the inverse of the one under HL conditions, as each odd win is more than offset by high-impact losses. Since the subordinates also experience a marked drop in their aggressive motivation, the fighting costs for the dominants are also indirectly reduced whenever they enter a fight against the subordinates. However, while the subordinates tend to participate in low-return fights, their winning probability nevertheless remains constant. Therefore, while subordinates reduce their costs of fighting by adjusting their fight dynamics, they at the same time retain the same overall probability to win future interactions. Though an immediate return of this mechanism may be minimal, given that the hierarchies are stable under such conditions, a long-term perspective may offer benefits to current subordinates who maintain a constant winning probability.

The results of the present study demonstrate that the low winner-high loser effect scenario resulted in a most favorable cost-benefit ratio of fights in a group irrespective of the final rank that an individual would achieve in the hierarchy. While proportional winner-loser effects also reduce the costs of fighting in groups, they do so more slowly and the costs are generally higher overall than under conditions featuring disproportionally high loser effects. However, increasingly different winning probabilities among individuals of different rank in scenarios with proportional winner-loser effects could mean that such conditions are beneficial under certain circumstances, such as cases when the costs of fighting are outweighed by the need to acquire, and distribute, resources at present. In contrast, with disproportionally low loser effects the subordinates are unable to conserve the resources by minimizing fighting costs, but could benefit from the potential to disrupt long-term hierarchical structure if the need for gains ends up overshadowing any costs incurred by fights. Prohibitive costs of such systems would, 
however, prevent this method of resource redistribution in a majority of situations. It is, thus, not surprising that examples in nature almost exclusively feature either proportional winner-loser effects or cases where loser effects are more pronounced.

\section{Acknowledgements}

We would like to thank current and past members of the Huber and Pavelic laboratories for their commentary on previous versions of this paper. We would also like to thank two anonymous reviewers for their helpful comments and suggestions.

\section{References}

Appleby, M.C. (1983). The probability of linearity in hierarchies. - Anim. Behav. 31: 600608.

Bakker, T.C.M., Bruijn, E. \& Sevenster, P. (1989). Asymmetrical effects of prior winning and losing on dominance in sticklebacks (Gasterosteus aculeatus L.). - Ethology 82: 224-229.

Beacham, J.L. (2003). Models of dominance hierarchy formation: effects of prior experience and intrinsic traits. - Behaviour 140: 1275-1303.

Beaugrand, J.P. \& Goulet, C. (2000). Distinguishing kinds of prior dominance and subordination experiences in males of green swordtail fish (Xiphophorus helleri). - Behav. Process. 50: 131-142.

Bergman, D.A., Kozlowski, C., McIntyre, J.C., Huber, R., Daws, A.G. \& Moore, P.A. (2003). Temporal dynamics and communication of winner-effects in the crayfish, Orconectes rusticus. - Behaviour 140: 805-825.

Bonabeau, E., Theraulaz, G. \& Deneubourg, J.L. (1996). Mathematical model of selforganizing hierarchies in animal societies. — Bull. Math. Biol. 58: 661-717.

Cant, M.A., English, S., Reeve, H.K. \& Field, J. (2006). Escalated conflict in a social hierarchy. - Proc. Roy. Soc. Lond. 273: 2977-2984.

Chase, I.D., Bartolomeo, C. \& Dugatkin, L.A. (1994). Aggressive interactions and intercontest interval - how long do winners keep winning. - Anim. Behav. 48: 393-400.

Chase, I.D. \& Rohwer, S. (1987). Two methods for quantifying the development of dominance hierarchies in large groups with applications to Harris' sparrows. - Anim. Behav. 35: 1113-1128.

Clutton-Brock, T.H., Albon, S.D., Gibson, R.M. \& Guinness, F.E. (1979). The logical stag: adaptive aspects of fighting in red deer (Cervus elaphus L.). - Anim. Behav. 27: 211225 .

Davis, K. \& Huber, R. (2007). Activity patterns, behavioural repertoires, and agonistic interactions of crayfish: a non-manipulative field study. - Behaviour 144: 229-247.

Dugatkin, L.A. (1997). Winner and loser effects and the structure of dominance hierarchies. - Behav. Ecol. 8: 583-587.

Dugatkin, L.A. \& Druen, M. (2004). The social implications of winner and loser effects. Proc. Roy. Soc. Lond. 271: 488-489. 
Dugatkin, L.A. \& Earley, R.L. (2003). Group fusion: the impact of winner, loser, and bystander effects on hierarchy formation in large groups. - Behav. Ecol. 14: 367-373.

Enquist, M. \& Leimar, O. (1983). Evolution of fighting behavior - decision rules and assessment of relative strength. - J. Theor. Biol. 102: 387-410.

Enquist, M. \& Leimar, O. (1987). Evolution of fighting behaviour: the effect of variation in resource value. - J. Theor. Biol. 127: 187-205.

Enquist, M., Plane, E. \& Roed, J. (1985). Aggressive communication in fulmars (Fulmarus glacialis) competing for food. - Anim. Behav. 33: 1007-1020.

Francis, R.C. (1988). On the relationship between aggression and social dominance. - Ethology 78: 223-237.

Goessmann, C., Hemelrijk, C.K. \& Huber, R. (2000). The formation and maintenance of crayfish hierarchies: behavioral and self-structuring properties. - Behav. Ecol. Sociobiol. 48: 418-428.

Grafen, A. (1987). The logic of divisively asymmetric contests — respect for ownership and the desperado effect. - Anim. Behav. 35: 462-467.

Hack, M.A. (1997). Assessment strategies in the contests of male crickets, Acheta domesticus (L). - Anim. Behav. 53: 733-747.

Hemelrijk, C.K. (2000). Towards the integration of social dominance and spatial structure. - Anim. Behav. 59: 1035-1048.

Hemelrijk, C.K. (2002). Understanding social behaviour with the help of complexity science. - Ethology 108: 655-671.

Hock, K. \& Huber, R. (2006). Modeling the acquisition of social rank in crayfish: winner and loser effects and self-structuring properties. - Behaviour 143: 325-346.

Hock, K. \& Huber, R. (2007). Effects of fighting decisions on formation and structure of dominance hierarchies. - Mar. Freshw. Behav. Physiol. 40: 153-169.

Hsu, Y.Y., Earley, R.L. \& Wolf, L.L. (2006). Modulation of aggressive behaviour by fighting experience: mechanisms and contest outcomes. - Biol. Rev. 81: 33-74.

Hsu, Y.Y. \& Wolf, L.L. (1999). The winner and loser effect: integrating multiple experiences. - Anim. Behav. 57: 903-910.

Hsu, Y.Y. \& Wolf, L.L. (2001). The winner and loser effect: what fighting behaviours are influenced? - Anim. Behav. 61: 777-786.

Huber, R. (2005). Amines and motivated behaviours: a simpler systems approach to complex behavioural phenomena. - J. Comp. Physiol. A 191: 231-239.

Huber, R., Daws, A., Tuttle, S.B. \& Panksepp, J.B. (2004). Quantitative behavioral techniques for the study of crustacean aggression. - In: Physiology of the crustacean nervous system (Wiese, K. \& Schmidt, M., eds). Springer, Berlin, p. 186-201.

Huber, R. \& Delago, A. (1998). Serotonin alters decisions to withdraw in fighting crayfish, Astacus astacus: the motivational concept revisited. - J. Comp. Physiol. A 182: 573583.

Huber, R. \& Kravitz, E.A. (1995). A quantitative analysis of agonistic behavior in juvenile American lobsters (Homarus americanus L). - Brain Behav. Evol. 50: 60-68.

Issa, F.A., Adamson, D.J. \& Edwards, D.H. (1999). Dominance hierarchy formation in juvenile crayfish Procambarus clarkii. - J. Exp. Biol. 202: 3497-3506.

Jackson, W.M. (1991). Why do winners keep winning? — Behav. Ecol. Sociobiol. 28: 271276.

Jameson, K.A., Appleby, M.C. \& Freeman, L.C. (1999). Finding an appropriate order for a hierarchy based on probabilistic dominance. - Anim. Behav. 57: 991-998. 
Jennings, D.J., Gammell, M.P., Carlin, C.M. \& Hayden, T.J. (2005). Win, lose or draw: a comparison of fight structure based on fight conclusion in the fallow deer. - Behaviour 142: 423-439.

Maynard Smith, J. (1982). Evolution and the theory of games. - Cambridge University Press, Cambridge.

Maynard Smith, J. \& Parker, G.A. (1976). The logic of asymmetric contests. — Anim. Behav. 24: $159-175$.

Mesterton-Gibbons, M. (1999). On the evolution of pure winner and loser effects: a gametheoretic model. - Bull. Math. Biol. 61: 1151-1186.

Morrell, L.J., Lindström, J. \& Ruxton, G.D. (2005). Why are small males aggressive? Proc. Biol. Sci. 272: 1235-1241.

Neat, F.C., Taylor, A.C. \& Huntingford, F.A. (1998). Proximate costs of fighting in male cichlid fish: the role of injuries and energy metabolism. - Anim. Behav. 29: 221-240.

Oyegbile, T.O. \& Marler, C.A. (2005). Winning fights elevates testosterone levels in California mice and enhances future ability to win fights. - Horm. Behav. 48: 259-267.

Pagel, M. \& Dawkins, M.S. (1997). Peck orders and group size in laying hens: 'future contracts' for non-aggression. - Behav. Process. 40: 13-25.

Panksepp, J.B. \& Huber, R. (2004). Ethological analyses of crayfish behavior: a new invertebrate system for measuring the rewarding properties of psychostimulants. - Behav. Brain Res. 153: 171-180.

Rutte, C., Taborsky, M. \& Brinkhof, M.W.G. (2006). What sets the odds for winning and losing? - Trends Ecol. Evol. 21: 16-21.

Schroeder, L. \& Huber, R. (2002). Fight strategies differ with size and allometric growth of claws in crayfish, Orconectes rusticus. - Behaviour 138: 1437-1449.

Schuett, G.W. (1997). Body size and agonistic experience affect dominance and mating success in male copperheads. - Anim. Behav. 54: 213-224.

Sokal, R.R. \& Rohlf, F.J. (1981). Biometry. - W.H. Freeman, New York, NY.

Stocker, A.M. \& Huber, R. (2001). Fighting strategies in crayfish Orconectes rusticus (Decapoda, Cambaridae) differ with hunger state and the presence of food cues. - Ethology 107: 727-736.

Thorpe, K.E., Taylor, A.C. \& Huntingford, F.A. (1995). How costly is fighting? Physiological effects of sustained exercise and fighting in swimming crabs, Necora puber (L.) (Brachyura, Portunidae). - Anim. Behav. 50: 1657-1666.

Van Doorn, G.S., Hengeveld, G.M. \& Weissing, F.J. (2003). The evolution of social dominance II: multi-player models. - Behaviour 140: 1333-1358.

Whitehouse, M.E.A. (1997). Experience influences male-male contests in the spider Argyrodes antipodiana (Theridiidae: Araneae). — Anim. Behav. 53: 913-923. 